\title{
Virilización total en una mujer: forma poco usual de presentación de la hiperplasia suprarrenal congénita
}

\author{
Dra. Ximena Cuello A.1; Dra. Nedy Abodowsky G.1, 2; Dra. Ximena Vivanco W. 1, 2; \\ Dr. Francisco Ossandón C.2,3

\section{Congenital adrenal hyperplasia presenting as total virilism in a 4 year old child}

4.5 year old patient presented with pubic hai, phallic enlargement, accelerated growth and no palpable gonads within the scrotum neicher the inguinal area. Nuclear chromatin was positive. Bone age was 7 years. In two separate $24 \mathrm{~h}$ urine collections, 17 cetosteroids and pregnanetriol were elevated. Serum 17 hydroxiprogesterone, $\Delta 4$ androstenedione, dehidroepiandrosterone and testosterone were also elevated, while deoxycorticosterone, corticosterone, follicle stimulating hormone and luteinizing stimulating hormone were nomal. Plasma cortisol did not increase after synacthen-rapid administration. Urinary adrenal steroid were supressed by dexamethasone. Pelvic ecothomography showed normal internal female genitalia. Non salt losing congenital adrenal hyperplasia was thus diagnosed. Histerectomy and oophorectomy were performed based on her psychological adaptation to male gender. (Key words: adrenal hyperplasia, congenital, non salt losing, virilism, puberty, precocius.)

La hiperplasia suprarrenal congénita (HSC) comprende una serie de alteraciones hereditarias de la esteroidogénesis, siendo el déficit de la 21 hidroxilasa (21-OH) la forma más frecuente de presentación y la causa más común de genitales ambiguos en la mujer.

El grado de virilización de los genitales externos se relaciona con la cuantia de la sobre. producción de andrógenos y el período del desarrollo fetal en que ésta se inicia.

En las niñas más severamente masculinizadas se pueden encontrar, excepcionalmente, estructuras prostáticas, fusión completa de labios y formación de uretra peneana, correspondiendo al grado $V$ de la clasificación de Prader ${ }^{1-4}$. Esta forma de presentación es rara: hemos encontrado 14 casos publicados en la literatura ${ }^{4-6}$, por lo que parece de interés presentar un paciente con dichas características.

1. Servicio de Pediatría, Hospital San Juan de Dios.

2. División Occidente, Facultad de Medicina, Universidad de Chile.

3. Servicio de Cingía Infantil, Hospital San Juan đe Dios.

\section{MATERIAL Y METODO}

En el paciente que se comunica se evaluaron peso y talla según los estándares de crecimiento de Tanner?, la edad ósea en radiografía de carpo y mano, segün Greulich y Pyle . $^{8}$

Se midieron 17 cetosteroides urinarios (17-Cs) por el método M.R.C. (Medical Research Council) Committee on Clinical Endocrinology $9 ; 17$ hjdroxycorticosteroides $(17-\mathrm{OH})$, por el método de Portet-Siver ${ }^{10}, 11$; compucsto tetrahidroesoxicortisol (THS), por una mođificación del método Porter-Silver ${ }^{12}$; dehidroepiandrosterona (DHA), por el método utilizado en el Servicio de Endocrinologia del profesor Bricaire, Hospital Cochiné, Paris 1980; cortisol libre urinario (FLU), medido por enzima inmunoensayo (EIA), por doble anticuerpo (Intnunotech Corp. Boston), pregnanetriol (PGT), por el método de Bongiovanni 13,14 y estrógenos totales $(\mathrm{EgT})$ por el método de Jayle 15

$\mathrm{Se}$ determinaran concentraciones plasmáticas de hormona folículo estimulante (FSH), hormona lutejnizante (LH), testosterona (T) dehidroepiandrosterona sulfato (DHAS) y 17-hidroxiprogesterona (17-OHP) por radioinmunoensayo.

En la Universidad de Cornell (USA) se realizaron determinaciones plasmáticas de 17-OHP, $\triangle 4$ androstenediona ( $\triangle 4$ ), DHAS, desoxicorticosterena (DOC), corticosterona (B) y cortisol (F).

Se realizó la estimulación suprarrenal con ACTH (Synacthen-rapid $0,25 \mathrm{mg}$ i.m.), se midieron concentraciones de $\mathbf{F}$ plasmático basal y a los 60 minutos, $17-0 H P$ basal, 30 y 60 minutos y prueba de frenado con dexametasona oral $2 \mathrm{mg}$ diarios por dos días, midiendo 
en orina de 24 horas $17 \mathrm{Cs}, 17-0 \mathrm{H}$, DHA, PGT y THS basales y después del estimulo.

Se realizó ecografía ginecológica, abdominal y ecotomografía suprarrenal.

\section{CASO CLINICO}

Pacjente de 4 años con fenotipo masculino. Consultó por desarrollo de genitales externos, aparición de vello pubiano y aceleración del crecimiento (tigura 1). Prjmer hijo de padres no colsangujneos. Antecedentes perinatales normales. Dos hermanas sanas, de diferentes padues.

A los dos años habia consultado en otro lugar por criptorquidea bilateral, que no se estudió.

Muy inquieto y agresivo. Peso $15 \mathrm{~kg}$, talla $113 \mathrm{~cm} \mathrm{(p}$ 97), presión arterial $90 / 60 \mathrm{~mm} \mathrm{Hg}$. Pente circuncidado de 7 cn de largo, meato urinario en el extremo del glande; escroto hjpodesarrollado, no pigmentado, sin gónadas palpables en su interior ni en las regiones inguinales. Desarrollo de vello pubiano en etapa III de Tanner.

Edad ósea 7 años, cromatina nuclear positiva (45\%). Catiograma 46 xx. Electrolitos plasmáticos normales, FSH: 1,7 UL/L; LH: 1,0 Ul/L; testosterona $198 \mathrm{ng} / \mathrm{dL}$; cortisol plasmático a las 8,00 AM. $8,6 \mathrm{ug} / \mathrm{dL}$ y a las $8: 00$ PM, 5,0 ug/dL. Determinaciones de metabolitos suprarrenales en orina de 24 horas, basales y después de dexametasona en tabla 1. Mediciones plasmáticas basales y despuéx de administrar ACTH, de 17-0HP y cortisol en tabla 2. Las determinaciones plasmáticas basales de la función suprarrenal realizadas en la Universidad de Cotnell, en New York, se describen en la tabla 3.

En la endoscopia uretro-vesical el aspecto do la uretra era masculino pormal, con vero montanum desatrollado; el orificio utricular tenía comunicación amplia con la vagina, cuello vesical y vejiga normales. La ecografía pelviana demostró presencia de útero.

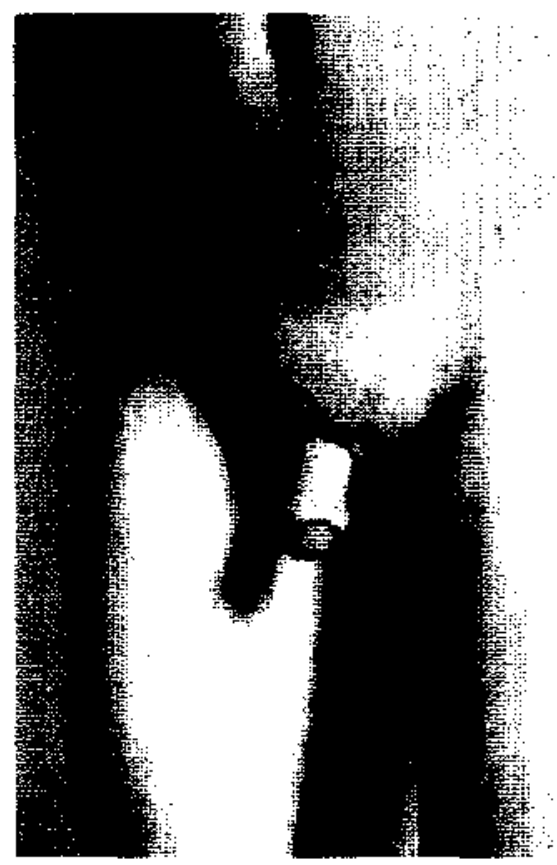

Figura 1: Paciente de sexo genético femenino, con masculinización completa de sus genitales externos.

Estos estudios permitieron hacer el diagnóstico de hiperplasia suprarrenal congénjta por falla de la 21-OH. Luego de evaluar la situación psiquiátrica, biológica y social de la paciente se practicó laparatomía media infraumbilical para extirpar gónadas y genitales internos.

Tabla 1

Eliminación en orina de $24 \mathrm{~h}$, bajo condiciones basales y de frenado con dexametasona (Dex) de los compuestos indicados

\begin{tabular}{|c|c|c|c|c|c|}
\hline \multicolumn{2}{|l|}{ Esteroide } & \multirow{2}{*}{$\begin{array}{r}\text { Basal } \\
25,8\end{array}$} & \multirow{2}{*}{$\begin{array}{c}\text { Valor normal } \\
\text { para la edad }\end{array}$} & \multirow{2}{*}{$\begin{array}{l}\text { Basal } \\
22,7\end{array}$} & \multirow{2}{*}{$\begin{array}{r}\text { Dex* } \\
6,3\end{array}$} \\
\hline $17-\mathrm{CS}$ & (mg/24 h) & & & & \\
\hline $17-\mathrm{OHcs}$ & $(\mathrm{mg} / 24 \mathrm{~h})$ & 3,0 & 3,0 & 4,3 & 0,0 \\
\hline DHA & $(\mathrm{mg} / 24 \mathrm{~h})$ & 0,5 & $<0,5$ & 0.6 & 0,3 \\
\hline PGT & $(\mathrm{mg} / 24 \mathrm{~h})$ & 7,3 & 0,0 & 7,9 & 1,1 \\
\hline THS & $(\mathrm{ug} / 24 \mathrm{~h})$ & $<20,0$ & $<500,0$ & $<20,0$ & $<20,0$ \\
\hline FLU & (ug/24h) & 22,0 & $30-150$ & - & - \\
\hline E. & $(\mathrm{ug} / 24 \mathrm{~h})$ & 34,0 & $<20,0$ & 0,0 & 0,0 \\
\hline Creatinina & $(\mathrm{mg} / 24 \mathrm{~h})$ & 336,0 & - & 207,0 & 300,0 \\
\hline
\end{tabular}

* 2 mg x 2 días.

$17 \mathrm{Cs}: 17$ cetosteroides.

DHA : dehidroepiandrosterona.

THS : tetrahidtodesoxicortisol.

E : estrógenos.
17 OHCS : 17 hidroxicorticosteroides.

PGT : pregnanotriol,

FLU : cortisol libre urinario. 
Ll estudio anatomopatológico confïrnò la ex stémalia de un útero de $4 \times 2 \times 0,6$ sms, euyo cuelk nedia 1 × $0,7 \mathrm{~cm}$, trompa derecha de $6 \times 0,3 \mathrm{~cm}$, ovarjo dereclon de $2,5 \times 0,8 \times 0,9 \mathrm{~cm}$, trotrpa izquierda de $6 \times 0,2$ เ ovario izquierdo de $2,2 \times 1,3 \times 0.7$ inn (1jgura 2 ).

Tabla 2

Concentracion plasmatica de 17 hidroxiprogesterona y cortisol en condiciones basales y dexpués de sstimulación con ACTH Symacthen rapid $(0,25 \mathrm{mg}$ i.r.

\begin{tabular}{|c|c|c|c|c|}
\hline & & Besill & 30 mín. & $6 \mathrm{U}$ riin \\
\hline (1) & $17 \mathrm{OHP}(\mathrm{Hg} / \mathrm{mL})$ & $>25^{*}$ & $>25^{*}$ &.- \\
\hline (2) & $\mathrm{F} \quad \quad(u g / d \mathbf{l})$ & 8,6 & - & 10 \\
\hline
\end{tabular}

(1) Valor normal para la edad: $0.2-1,8 \mathrm{ng} / \mathrm{mL}$

(2) Valor normal para la edad: $6,5-26 \mathrm{ug} / \mathrm{dL}$.

Limitio máximo de concentración de la curva de da. boratotio.

17OHP: 17 hidroxiprogesterona, f': costivol.

Tabla 3

Parámetros plasmátioos basales de la función suprarrenal

\begin{tabular}{|c|c|c|c|}
\hline & & Basal & Kaugo normal* \\
\hline DOC & $(n g / d L)$ & 46,4 & $(2-87)$ \\
\hline B & $(\mathrm{ug} / \mathrm{dL})$ & 0,855 & $(0,06 \cdot 3,0)$ \\
\hline F & $\left\{u_{g} / d L\right\}$ & 1,7 & $(5-32)$ \\
\hline$\Delta 4$ & $\left(\mathrm{n}_{g} f \mathrm{dL}\right)$ & $1.361,0$ & $(3-63)$ \\
\hline DHFA & (ng/dL) & 292,0 & $(5-287)$ \\
\hline $\mathrm{T}$ & $(\mathrm{ng} / \mathrm{dL})$ & 121,0 & $(2-12)$ \\
\hline $170 \mathrm{OH}$ & (nginL) & $4.006,0$ & $(0,07-1,4)$ \\
\hline $17 \triangle 5 P$ & (ng;dl) & 669.0 & (21-168) \\
\hline
\end{tabular}

* Pang S., Levine L.S., Stoner E., et al, (27).

DOC: desoxicortidosterona. 13: corticosterona. $\Gamma$ : cortisol. $\triangle 4: \triangle 4$ androstenidona. Gilks: delidroepiandrosterona. $T$ : testosterona. 17 OHP: 17 hidroxiprog LSterona. $17 \triangle 5 \mathrm{P}$ : pregnenolena.

\section{DISCUSION}

Los hechos clinicos más llamativos en esta paciente, con fenotipo masculino, fueron la apdrición de signos de virilización a una clad temprana y la ausencia de testículos palpables. La existencia de pubertad precoz verdadera o de tumor productor de gonadotrofinas se descarto por las concentraciones normales de FSH y LHy

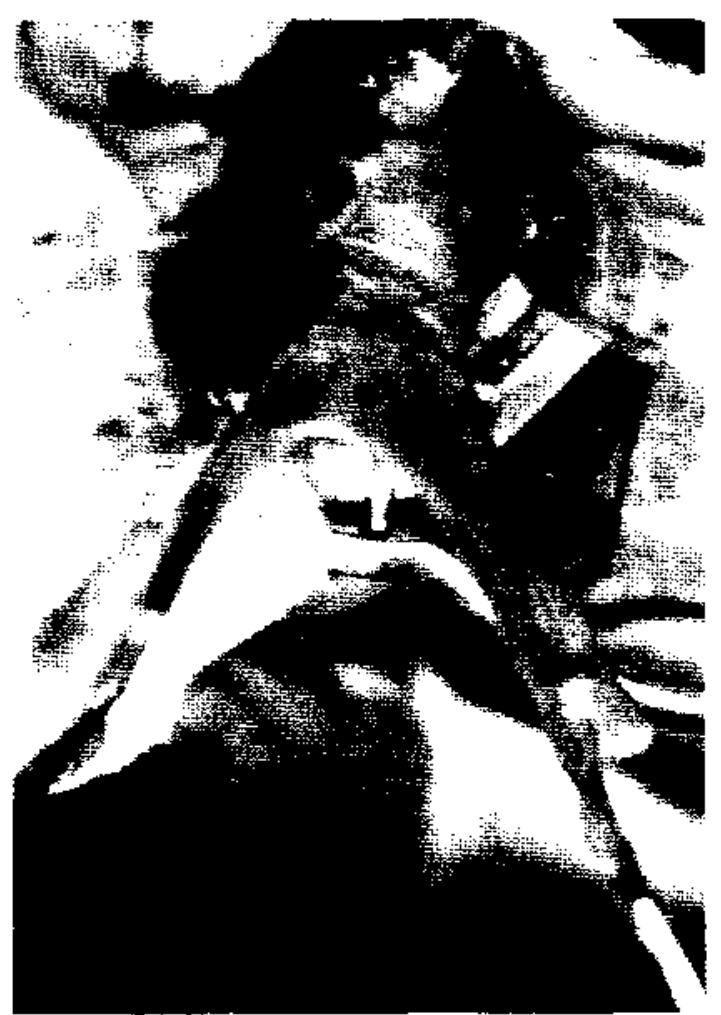

Figura 2: Pieza opcratoria de los genitales internus, ítero, trompa y ovarios.

marcadores tumorales negativos (hC, sub $U \beta$ y $\alpha$ fetoproteina).

La existencia de cromatina nuclear positiva $y$ carotipo $46 \mathrm{xx}$ indujo a pensar que su sexo era femenino, con masculinización extrema de los genitales externos. Esto fue confirmado por el aumento de los 17-Cs y PGT en orina de $24 \mathrm{~h}$; de T. 17-OHP, DHAS y $\Delta 4$ en plasma, respuesta débil del cortisol frente a la estimulación con ACTH y los genitales internos femeninos detectados en la ecotomografía.

El defecto enzimático correspondía a falla de 21-OH, que además de ser la forma thás comútı, se caracteriza por cifras muy aumentadas de [7-OHP y bajas de cortisol después del estímulo con ACTH. Nuestro paciente no sería perdedor de sal, pues tienc concentraciones normales de DOC y $\mathbf{B}$, allnque no pudimos medir actividad de renina plasmática para confirmarlo.

Dos hechos contribuyeron a la tardanza en identificar su problema: la total masculinización de los genitales externos, que es rara, y la ausencia de pérdidas de sal. 
Encontramos 14 casos descritos hasta el año 1969: 7 se diagnosticaron en lá autopsia, sólo 2 no eran perdedores de sal ${ }^{4-6}$. Dentro de esta casuística, Prađer describió 7 niños con uretra fálica completa; todos eran perdedores de $\mathrm{sal}^{6}$.

No encontramos publicaciones posteriores a septiembre de 1987 de casos similares ${ }^{17,18}$.

Para explicar la extensa virilización observada en nuestro paciente, debemos suponer que el feto fue expuesto, en el período critico de la diferenciación sexual ( 10 a 12 semanas), a subreproducción de andrógenos adrenales, los que produjeron profunda masculinización de los genitales externos, incluyendo el desarrollo de la uretra peneana. El cariotipo $46 \mathrm{xx}$ y la ausencia de testículos fetales funcionantes normales explican el desarrollo de los genitales internos fe. meninos.

E1 largo tiempo de evolución de su afección sin tratamiento nos llevó a estudiar la posibilidad de asociación con un tumor suprarrenal, ya que se postula como mecanismo etiopatogénico la hiperestimulación mantenida con $\mathrm{ACTH}^{20}$. La mayoría de estos tumores se hacen ACTH independientes y sólo algunos son frenados con deXametasona ${ }^{19}$. Por lo tanto, esta prueba por sí sola no es suficiente para excluir la posibilidad de tumor de pacientes con HSC, para lo que sería necesario ampliar el estudio de las glándulas suprarrenales con ecografía y tomografía axial computada.

En nuestro paciente se obtuvo frenado con dexametasona, la ecografía suprarrenal fue normal $y$ durante el acto quirúrgico se exploraron las glándulas suprarrenales, encontrándoselas macroscópicamente normales. Se descartó así la asociación de HSC con adenoma suprarrenal.

Por otra parte, es notable la existencia de un afecto androgénico tan acentuado, sin pérdida de sal. Esto podria explicarse fisiopatológicamente basándose en lo propuesto por New y Sea$\operatorname{man}^{2}, 22$, quienes señalan que la zona glomerulosa y la zona fasciculata funcionan como dos glándulas separadas con respecto a su regulación y secreción. De acuerdo con este concepto, la biosíntesis esteroidal de la zona "fasciculata" es regulada por $\mathrm{ACTH}$, mientras la glomerulosa lo es por el sistema renina-angiotensina: el ACTH sólo influiría secundariamente en la secreción glome rular de aldosterona.

Las consecuencias del diagnóstico tardío, con desenlace fatal, en los niños perdedores de sal, ha sido bien documentada en otras publicaciones ${ }^{24}$. En pacientes no perdedores de sal y sin malformaciones genitales, la asignación correcta de] sexo en una edad temprana constituye un problema cuya solución es urgente. Esto ha llevado, a diferentes investigadores, a plantear la posibilidad de hacer exámenes de rastreo en el período neonatal para HSC, con el objeto de realizar precozmente el diagnóstico y dar tratamiento oportuno, lo que evitaria muertes prematuras, errores en la asignación del sexo, virilización progresiva que resultc en prematura fusion de las epifisis y eventual baja estatura ${ }^{24-26}$.

En nuestro caso, genéticamente femenino, debido a la presencia de un desorden intersexual que se hizo evidente sólo en la edad preescolar tardía, en una mujer severamente masculinizada, que había sido criada como varón y que psicológicamente estaba adaptada a dicho rol, se decidió dejarla con sexo masculino ${ }^{28}$, extirpando sus genitales internos, para hacerle, en el futuro, tratamiento sustitutivo con testosterona e instalarle prótesis testiculares.

\section{RESUMEN}

Un paciente de cuatro ar̃os y medio consultó por aparición de vello pubiano, crecimiento peneano, aceleración del crecimiento, los testículos no se palpaban en escroto ni en áreas inguinales. Cromatina nuclear positiva. Edad ósea 7 años. 17 cetosterojdes y pregnanetriol aumentados en 2 recolecciones separadas de orina de $24 \mathrm{~h}$. Tambièn estahan elevadas las concentraciones plasmáticas de 17 hidroxiprogesterona, $\Delta 4$ androstenediona, dehidroepiandrosterona $y$ testosterona, mientras que las de desoxicorticosterona, corticosterona, FSH y LH eran normales. E1 cortisol plasmático no aumentó después de estimulación con ACTH. Los esteroides urinarios suprarrenales fueron frenados con dexametasona. La ecotomografía pelviana demostró que tenía genitales internos femeninos normales. Se con. cluyó finalmente que se trataba de un caso de hiperplasia suprarrenal congénita no perdedora de sal, diagnosticada en forma tardía. Se practicó histerectomia y ooforectomía, basándose en su buena adaptación psicológica al sexo masculino de crianza. 


\section{AGRADECIMIENTOS}

Se agradece a la Dra. María New de la Universidad de Cornel, EE.UU., las mediciones plasmáticas de 17 OHP, $\triangle 4$ androstenediona, DHAS, DOC, B y $\mathrm{F}$.

\section{REFERENCIAS}

1. Rappoport R., Claive Nihoul-Fekete: Pseudo heymafrodisme féminin. Etiologie et phisiopathologie. Ann Endocrinol (Paris) 1980; $41 ; 345-353$.

2. New M.L.: Clinical and endocrinological aspects of 21.hidroxilase deficiency. Ann NY Acad Sci 1985; 458: 1-27.

3. Wilkins L.: Adrenal Disorders II. Congenital virilizing adrenal hiperplasia. Arch Dis Child 1962; 37 ; 231-241.

4. Bentinck R.C., I,isser H. Reilly W.A.: Female pseudohermaphrodism with penileuretra, masquerading as precocius puberty and cryptorchidism. J Clin Endocrinol Metab 1959; $16: 412-418$.

5. Rosenberg B., Hendren W.H., Gawford J.D.: Posterior urethrovaginal communication in apparent males with congenital adrenocortical hiperplasia. $N$ Engl J Med 1969; 280: 131-134.

6. Maxied W., Baker $R$, McChystal $H$, Fitzgerald E.: Complete masculinization of the external genitalia in congenital adrenocortical hiperplasia, presentation of two cases. J Urolog 1965; $94: 266-270$.

7. Tanner J.M.. White House R.H., Takaishi M. Standards from birth to maturity for height, weight, height velocity and weight velocity: British children 1965. Arch Dis Child 1966; 41: 220-613.

8. Grewich W., Pyle S.L.: Radiographic Atlas of Ske1etal development of the hand and wrist. 2nd Ed, Stanford, Stanford University Press, and London Oxford University Press. 1959.

9. Proposed method for 17.cetoesteroids determination: Lancet $1951 ; 2$ : $\$ 85$.

10. Silber R.M., Porter C.C.: The determination of 17 , 21-dehidroxy -20-ketosteroids in urine and plasm. J Biol Chem 1954; 210: 923-982.

11. Votgt K.D.: Determination of 17-hidroxicorticosteroids in urine as Porter-Silber Chromogens in "Method of Hormone Analysis" Stuttgart, Germany, Brewer H., Hanel D., Koirs Kenpertt. 1976.

12. Revol A., Plasse J.C., Asthier J., Piccard G.: Controle de qualité en Hormonologie steroide. Gentileza Div. Laboratorios bio Mérieux, Paris 1971.

13. Bongiovanni A.M., Eberlein W.R.: Critical Analysis of Methods of meassurement of pregnane-3-Alpha,
17-Alpha, 20-Alphatriol in human orine. Anal Chem 1958; $30: 388$.

14. Salazar M. Gacitúa $E$.: Niveles de pregnanetriol urinario en la hiperplasia virilizante y en el S.OP. Tesis. Facultad de Ciencias Químicas, 1977.

15. Jayle M.F., Crespy $O_{1}$ : Bulletin de la Socjété de Chimie Biologique 1950; 32: 1067-1071.

16. Mauvais-Jarvis P., Sitrik-Ware R., Labrie F.: Medicine de la reproduction, ginecologie endocrinienne. Cap. 16 Les anomalies de la différencjation sexuelle. Presses de la Université de Montreal.

1?. Vivanco $X$., Telterias $L .$, Abodousky $N$., Cuello $X$.: Hiperplasia suprattenal virilizante. Revisión de 23 años (1963-1986). Bol, Hosp. Sn. J. de Dios 1987; 34(3): 158-161.

18. Jarrea F., Ulloa A., Aguine y Pérez-Palacios $G_{\text {.: }}$ Hiperplasia suprarrenal congénita como causa de pseudohemafroditismo femenino. Rev Invest Clín (Méx). 1986; 38: 209-217.

19. Rayfield E.J., Rose L.J, Cain J.P., Dluhy R., Williams $G$ : ACTH-Responsive, dexamethasone-suppressible adrenocortical carcinoma. N Engl J Med 1971; 284: 591 .

20. Pang S. Becker D., Cotelingam J., Fotey T.P., Jr. and Drash A.L.: Adrenocortical tumor in a patient with congenital adrenal hiperplasia due to $21-\mathrm{Hi}$ droxilasa deficiency. Pediatrics 1981; 68(2): 242-246.

21. Phillip D.X. Lee, Winter R.J., Green O.C.: Virilizing adrenocortical tumors in childhood: eight cases and a review of the literature. Pediatrics $1985,76(3): 437$.

22. Savage: Congenital adrenal hiperplasia. Clin Endoc Metab 1985; 14(4): 893-909.

23. Bongiovanni A.M.. Root A.W.: The adrenogenital syndrome. N Engl J Med 1963; 268: $1283-1288$.

24. Lebovitz R.M., Pauli R.M. Laxove R.: Delayed diagnosis in congenital adrenal hiperplasia. Need for newborn screening. Am I Dis Child 1984; 138: 571.573 .

25. Pang $S$, Spence $D . A .$, New M.I, Newborn screening for congenital adrenal hiperplasia with special reference to scteening in Alaska. Ann NY Acad Sci $1985 ; 485: 90-102$.

26. Kuirdi $N$. et al.: Should we screen for congenital adrenal hiperpisia? A review of 117 cases. Arch Dis Child 1987, 62: 659-662.

27. Pang S., Levine L.S, Stoner E., Opitz J.M., Pollack M.S., Dupont B., New M.I.: Nonsalt-losing congenital adrenal hiperplasia due to $3-\beta$-hydroxy steroid dehydrogenose deficiency with narmal glomerulosa function. J Clin Endoctinol Metab 1983; 56: 808-818.

28. Dewhurst J., Grand D.B.: Intersex problems. Arch Dis Child 1984; $59: 1191-1194$. 